Ein Modalmaß für Beobachtbarkeit und perspektivische Beobachtbarkeit linearer zeitinvarianter Systeme

Autoren: Richard Seeber, Nicolaos Dourdoumas, Martin Horn

Journal: at - Automatisierungstechnik

DOI: 10.1515 /auto-2017-0015

Die Verlagsveröffentlichung ist verfügbar unter www. degruyter.com bzw. unter https://dx.doi.org/10.1515/auto-2017-0015 


\title{
Methoden
}

Richard Seeber*, Nicolaos Dourdoumas und Martin Horn

\section{Ein Modalmaß für Beobachtbarkeit und perspektivische Beobachtbarkeit linearer zeitinvarianter Systeme}

\author{
A modal measure for observability and perspective observability of linear time-invariant \\ systems
}

https://doi.org/10.1515/auto-2017-0015

Eingang 20. Februar 2017; angenommen 14. August 2017

Zusammenfassung: Das Modalmaß nach Litz stellt eine einfache Möglichkeit zur quantitativen Bewertung der Beobachtbarkeit eines Systems dar. Einen durch mehrfache Eigenwerte verursachten Beobachtbarkeitsverlust zeigt es jedoch mitunter nicht korrekt an. Vorliegender Beitrag schlägt eine Erweiterung vor, welche dieses Problem behebt. Im Weiteren wird gezeigt, dass sich das modifizierte Maß leicht auf die perspektivische Beobachtbarkeit erweitern lässt. Die praktische Anwendbarkeit des erweiterten Maßes wird anhand eines anschaulichen Beispiels demonstriert.

Schlüsselwörter: Beobachbarkeitsmaß, Modalmaß, perspektivische Beobachtbarkeit.

Abstract: The modal measure due to Litz is a simple method for quantitatively assessing a system's observability. In some cases, however, it does not correctly reflect the loss of observability caused by eigenvalues with multiplicities greater than one. The present contribution proposes an extension that remedies this problem. It is furthermore shown that this modified measure can easily be extended to assess also a system's perspective observability. The practical applicability of the measure is demonstrated in the course of an illustrative example.

*Korrespondenzautor: Richard Seeber, Institut für Regelungsund Automatisierungstechnik, Technische Universität Graz, Inffeldgasse 21/B/I, $8010 \mathrm{Graz,} \mathrm{Österreich,}$

E-Mail: richard.seeber@tugraz.at

Nicolaos Dourdoumas, Martin Horn: Institut für Regelungsund Automatisierungstechnik, Technische Universität Graz, Inffeldgasse 21/B/I, $8010 \mathrm{Graz}$, Österreich
Keywords: Observability measure, modal measure, perspective observability.

\section{Einleitung}

Dieser Beitrag befasst sich mit linearen zeitinvarianten Systemen der Form

$$
\frac{\mathrm{dx}}{\mathrm{d} t}=\mathbf{A x}, \quad \mathbf{y}=\mathbf{C x}
$$

Dabei bezeichnen die reellwertigen Matrizen $\mathbf{A} \in \mathbb{R}^{n \times n}$ und $\mathbf{C} \in \mathbb{R}^{m \times n}$ die System- bzw. die Ausgangsmatrix. Die Vektoren $\mathbf{x}$ und $\mathbf{y}$ stellen den Zustands- resp. Ausgangsvektor dar. Der Anfangszustand wird mit $\mathbf{x}_{0}:=\mathbf{x}(t=0)$ bezeichnet.

Betrachtet werden Beobachtbarkeitseigenschaften dieses Systems: einerseits die klassische Beobachtbarkeit nach Kalman, andererseits die sogenannte perspektivische Beobachtbarkeit, die in [6] eingeführt wird. Letztere ist, wie später gezeigt wird, u.a. für Aufgaben aus der Bildverarbeitung relevant. Für ihre formale Definition werden die sogenannten homogenen Koordinaten $[y]$ eines Vektors y benötigt. Diese sind für von Null verschiedene Vektoren $\mathbf{y}_{1}, \mathbf{y}_{2}$ durch die Äquivalenzrelation

$$
\left[\mathrm{y}_{1}\right]=\left[\mathrm{y}_{2}\right] \quad \Leftrightarrow \quad \frac{\mathbf{y}_{1}}{\left\|\mathbf{y}_{1}\right\|}=\frac{\mathbf{y}_{2}}{\left\|\mathbf{y}_{2}\right\|}
$$

definiert. Kenntnis von $[\mathbf{y}]$ bedeutet also Kenntnis der Richtung des Vektors y, nicht aber seiner Länge.

Definition 1. Das System (1) heißt perspektivisch beobachtbar, wenn aus Kenntnis des Funktionsverlaufs [y $(t)]$ auf einem endlichen Zeitintervall $t \in[0, T]$ mit $T>0$ der Anfangszustand in homogenen Koordinaten $\left[\mathbf{x}_{0}\right]$ eindeutig rekonstruierbar ist. 
Grob gesprochen bedeutet perspektivische Beobachtbarkeit also Rekonstruierbarkeit der Richtung des Anfangszustands aus der Richtung des Ausgangsvektors.

Zur Überprüfung der Beobachtbarkeit dient u. a. das Popov-Belevich-Hautus Kriterium. Nach diesem ist das System (1) genau dann beobachtbar, wenn für alle Zahlen $\lambda$ aus den Eigenwerten $s_{1}, \ldots, s_{n}$ der Systemmatrix A die sogenannte Hautus-Matrix

$$
\mathbf{H}_{\mathrm{b}}(\lambda):=\left[\begin{array}{c}
\lambda \mathbf{E}-\mathbf{A} \\
\mathbf{C}
\end{array}\right]
$$

vollen Rang $n$ aufweist. Dabei bezeichnet $\mathbf{E}$ die Einheitsmatrix. In [3] wird gezeigt, dass für die perspektivische Beobachtbarkeit der Rang einer ähnlich aufgebauten, sogenannten erweiterten Hautus-Matrix

$$
\mathbf{H}_{\mathrm{p}}\left(\lambda_{1}, \lambda_{2}\right):=\left[\begin{array}{c}
\left(\lambda_{1} \mathbf{E}-\mathbf{A}\right)\left(\lambda_{2} \mathbf{E}-\mathbf{A}\right) \\
\mathbf{C}
\end{array}\right]
$$

für alle Paare $\lambda_{1}, \lambda_{2}$ zweier Eigenwerte der Matrix A ausschlaggebend ist.

Diese Kriterien erlauben prinzipiell nur eine binäre Aussage; eine quantitative Beurteilung der Eigenschaften, d. h. eine Beantwortung der Frage, wie „gut“ (perspektivisch) beobachtbar ein System ist, ist mit ihnen nicht möglich. Letztere ist aber gerade für die Praxis von großer Bedeutung, z. B. wenn für ein gegebenes System aus einer Reihe von Sensorkonfigurationen die beste ausgewählt werden soll.

Dieses Problem, die Bewertung der Eigenschaften Beobachtbarkeit und perspektivische Beobachtbarkeit, ist Gegenstand des vorliegenden Beitrags. Im Falle der Beobachtbarkeit werden dazu in der Literatur verschiedene Möglichkeiten in Form sogenannter Beobachtbarkeitsmaße bzw. Steuerbarkeitsmaße vorgeschlagen, siehe z. B. [14] für einen Überblick. Letztere lassen sich aufgrund der Dualität der Eigenschaften unmittelbar auf die Beobachtbarkeit übertragen.

Ein solches Maß ist das in [16] eingeführte Distanzmaß. Dieses ist durch die Norm der kleinsten Störung gegeben, die zu einem Beobachtbarkeitsverlust führt, d. $h .^{1}$

$$
\mu:=\min _{\delta \mathbf{A}, \delta \mathbf{C}}\left\{\left\|\left[\begin{array}{l}
\delta \mathbf{A} \\
\delta \mathbf{C}
\end{array}\right]\right\|_{2} \mid(\mathbf{A}+\delta \mathbf{A}, \mathbf{C}+\delta \mathbf{C}) \text { nicht beob. }\right\} .
$$

1 Es bezeichnet $\|\mathbf{M}\|_{2}$ die Spektralnorm, d.h. den größten Singulärwert der Matrix M.
Es lässt sich durch Minimieren des kleinsten Singulärwertes der Matrix $\mathbf{H}_{\mathrm{b}}(\lambda)$ bezüglich $\lambda$ ermitteln [5]. In [15] wird zur Vermeidung numerischer Probleme bei der Überprüfung der perspektivische Beobachtbarkeit eine geradlinige Erweiterung dieses bereits sehr aufwändigen Optimierungsproblems vorgeschlagen. Man erhält dabei jedoch kein Distanzmaß für die perspektivische Beobachtbarkeit; für dieses ergibt sich eine noch deutlich kompliziertere Optimierungsaufgabe [19].

Eine mögliche Alternative stellen sogenannte Modalmaße dar, welche die Möglichkeit zur getrennten Beurteilung der Beobachtbarkeit einzelner Eigenwerte (Modi) der Matrix A bieten. Im Vergleich zum Distanzmaß zeichnen sich diese in der Regel durch eine einfachere Berechnungsvorschrift aus. Damit sind sie besonders gut geeignet, wenn z. B. im Rahmen von Parameterstudien unterschiedliche Sensor- oder Aktuatorkonfigurationen miteinander verglichen werden sollen, siehe z. B. [17, 18, 20, 21].

Beim Modalmaß nach Litz [12], welches auf einem Vorschlag von Lückel und Müller [10] basiert, wird dazu das Produkt von Ausgangsmatrix C und den Eigenvektoren von $\mathbf{A}$ herangezogen. In der englischsprachigen Literatur wird zu einem späteren Zeitpunkt ein ähnliches Maß von Hamdan und Nayfeh [8] vorgeschlagen sowie u. a. auch in $[9,11,13]$ betrachtet. Seltener verwendet wird dagegen ein Ansatz von Tarokh [23], der die Bewertung der Beobachtbarkeit anhand des Zählers der Übertragungsmatrix vom Systemzustand zum Ausgang vorschlägt. Beide Ansätze lassen sich anhand der Modalform (Diagonalform) des Systems anschaulich deuten. Sie weisen jedoch auch Schwachstellen auf.

Bevor auf diese näher eingegangen werden kann, wird zunächst die Bedeutung der Begriffe Beobachtbarkeitsmaß und modales Beobachtbarkeitsmaß konkretisiert. Unter ersterem wird im Rahmen dieses Beitrags Folgendes verstanden (in der Literatur wird der Begriff mitunter liberaler verwendet):

Definition 2. Eine Funktion $\mu(\mathbf{A}, \mathbf{C})$ heißt Beobachtbarkeitsmaß, wenn sie folgende Bedingungen erfüllt:

1. Nichtnegativität: $\mu(\mathbf{A}, \mathbf{C}) \geq 0$

2. Konsistenz: $\mu(\mathbf{A}, \mathbf{C})=0 \Leftrightarrow(\mathbf{A}, \mathbf{C})$ nicht beobachtbar 3. Stetigkeit: $\mu(\mathbf{A}, \mathbf{C})$ ist stetig in $\mathbf{A}, \mathbf{C}$.

Mitunter ist außerdem die Invarianz des Maßes unter bestimmten (z. B. orthogonalen) Zustandstransformationen wünschenswert. Obige Definition ist aus praktischer Sicht vernünftig: Die Konsistenz garantiert, dass ein Verlust der Beobachtbarkeit durch den Wert Null angezeigt wird, während die Stetigkeit sicher stellt, dass der Übergang zu Null nicht sprunghaft erfolgen kann. Unter einem modalen Beobachtbarkeitsmaß wird Folgendes verstanden: 
Definition 3. Eine Funktion $v_{\lambda}(\mathrm{A}, \mathrm{C})$ heißt modales Beobachtbarkeitsmaß, wenn sie für Eigenwerte $\lambda$ von $\mathbf{A}, \mathrm{d} . \mathrm{h}$. für $\operatorname{det}(\lambda \mathbf{E}-\mathbf{A})=0$, folgende Bedingungen erfüllt:

1. Nichtnegativität: $v_{\lambda}(\mathbf{A}, \mathbf{C}) \geq 0$.

2. Konsistenz: $v_{\lambda}(\mathbf{A}, \mathbf{C})=0 \Leftrightarrow \operatorname{rang} \mathbf{H}_{\mathrm{b}}(\lambda)<n$, d. h. der Eigenwert $\lambda$ ist nicht beobachtbar.

3. Stetigkeit: $v_{\lambda}(\mathbf{A}, \mathbf{C})$ ist stetig in $\mathbf{A}, \mathbf{C}, \lambda$.

Das Minimum

$$
v(\mathbf{A}, \mathbf{C}):=\min _{k} v_{s_{k}}(\mathbf{A}, \mathbf{C})
$$

eines Modalmaßes bezüglich der Eigenwerte $s_{1}, \ldots, s_{n}$ von A ist damit ein Beobachtbarkeitsmaß.

Die zuvor erwähnten Schwachstellen der Maße nach Litz bzw. Tarokh treten im Zusammenhang mit Systeme mit mehrfachen Eigenwerten auf. Die Berechnung der beiden Maße für solche Systeme wird zwar in [10] bzw. [23] diskutiert, jedoch unter Verwendung von Fallunterscheidungen. Beim Übergang von einfachen zu mehrfachen Eigenwerten sind die Maße dadurch mitunter nicht stetig, sodass es sich streng genommen nicht um Modalmaße im Sinne von Definition 3 handelt. Formal lässt sich dieser Umstand zwar dadurch beheben, dass die Maße nur für Systeme mit einfachen Eigenwerten definiert werden; wie anhand eines Beispiels eindrucksvoll gezeigt werden wird, führt dies aber nicht unbedingt zu sinnvollen Ergebnissen.

Motiviert durch diesen Umstand stellt der vorliegende Beitrag zunächst eine Erweiterung des Maßes nach Litz zu einem konsistenten und stetigen Modalmaß für die Beobachtbarkeit vor. Anschließend wird davon ausgehend ein Modalmaß für die perspektivische Beobachtbarkeit vorgeschlagen. Dazu werden in Abschnitt 2 kurz die beiden erwähnten Modalmaße näher erläutert und anhand eines Beispiels die diskutierten Schwachstellen gezeigt. Anschließend wird die genannte Erweiterung für die Beobachtbarkeit diskutiert. Wichtige Eigenschaften des vorgeschlagenen Maßes, insbesondere dessen nicht unmittelbar ersichtliche Stetigkeit, sowie eine Methode zu dessen Abschätzung bei ungenau bekannten Systemdaten werden diskutiert. In Abschnitt 3 wird die Eigenschaft perspektivische Beobachtbarkeit anhand eines Beispiels veranschaulicht und das bereits erwähnte Kriterium für die perspektivische Beobachtbarkeit erläutert. Dann wird ein Modalmaß für diese Eigenschaft vorgestellt und dessen Berechnung demonstriert. Abschnitt 4 schließlich gibt eine Zusammenfassung des Beitrags an.

\section{Modales Beobachtbarkeitsmaß}

Es werden zunächst zwei existierende Vorschriften zur Berechnung von Maßzahlen diskutiert. Dabei wird gezeigt, dass diese Definition 3 prinzipbedingt nicht erfüllen können. Anschließend wird ein neues Maß vorgeschlagen, welches diese Schwachstellen nicht aufweist, und dessen Berechnung anhand eines Beispiels demonstriert. Wichtige Eigenschaften dieses Maßes, u. a. Konsistenz und Stetigkeit, werden gezeigt. Schließlich wird im Rahmen eines Beispiels die Möglichkeit zur Abschätzung des Maßes bei ungenau bekannten Systemdaten diskutiert.

\subsection{Existierende Maßzahlen}

Zur Bewertung der Beobachtbarkeit des Eigenwertes $\lambda$ der Matrix A mit zugehörigem Eigenvektor p schlägt Litz in [12] folgende Maßzahl vor:

$$
\kappa_{\lambda}(\mathbf{A}, \mathbf{C}):=\frac{\mathbf{p}^{\mathrm{H}} \mathbf{C}^{\mathrm{T}} \mathbf{C} \mathbf{p}}{\mathbf{p}^{\mathrm{H}} \mathbf{p}} \operatorname{mit}(\lambda \mathbf{E}-\mathbf{A}) \mathbf{p}=\mathbf{0} .
$$

Darin bezeichnet $\mathbf{p}^{\mathrm{H}}$ den konjugiert komplexen und transponierten Vektor p. Die von Tarokh in [23] vorgeschlagene Maßzahl ist durch

$$
\begin{aligned}
\xi_{\lambda}(\mathbf{A}, \mathbf{C}): & =\left\|\mathbf{C}(\lambda \mathbf{E}-\mathbf{A})^{-1} \operatorname{det}(\lambda \mathbf{E}-\mathbf{A})\right\|_{2} \\
& =\|\mathbf{C} \operatorname{adj}(\lambda \mathbf{E}-\mathbf{A})\|_{2}
\end{aligned}
$$

gegeben. Bei diesen Ausdrücken wird jeweils vorausgesetzt, dass A einfache Eigenwerte besitzt; für die Vorgangsweise bei mehrfachen Eigenwerten sei auf [10] bzw. [23] verwiesen.

Man kann folgenden Zusammenhang der beiden Maße zeigen: Bezeichnen $\sigma_{1} \geq \sigma_{2} \geq \ldots \geq \sigma_{n}=0$ die Singulärwerte der Matrix $\lambda \mathbf{E}-\mathbf{A}$, so gilt

$$
\xi_{\lambda}=\sqrt{\kappa_{\lambda}} \cdot \prod_{i=1}^{n-1} \sigma_{i} .
$$

Die erwähnten Schwachstellen der beiden Maße werden anhand eines Beispiels demonstriert. Für das folgende System mit zwei möglichen Ausgangsmatrizen $\mathbf{C}_{1}$ und $\mathbf{C}_{2}$

$$
\mathbf{A}=\left[\begin{array}{cc}
1 & 0 \\
0 & 1+\alpha
\end{array}\right], \quad \mathbf{C}_{1}=\left[\begin{array}{ll}
1 & 1
\end{array}\right], \quad \mathbf{C}_{2}=\mathbf{E}
$$

lauten die Eigenwerte $s_{1}=1$ und $s_{2}=1+\alpha$; unter der Voraussetzung $\alpha \neq 0$ erhält man sowohl für $\mathbf{C}=\mathbf{C}_{1}$ als auch für $\mathbf{C}=\mathbf{C}_{2}$ (!) die Maßzahlen

$$
\begin{aligned}
& \kappa_{s_{1}}=\kappa_{s_{2}}=1 \\
& \xi_{s_{1}}=\xi_{s_{2}}=|\alpha| .
\end{aligned}
$$


Für $\mathbf{C}=\mathrm{C}_{1}$ hat $\alpha$ offensichtlich einen Einfluss auf die Beobachtbarkeit, da $\left(\mathbf{A}, \mathbf{C}_{1}\right)$ für $\alpha=0$ nicht beobachtbar ist. Dennoch sind die Maßzahlen nach Litz unabhängig von $\alpha$ und verschieden von Null! Deren konsistente Festlegung für $\alpha=0$ führt in diesem Fall somit zwangsläufig zu einer Unstetigkeit. Für $\mathbf{C}=\mathbf{C}_{2}$ ist der Zustand vollständig messbar, sodass $\alpha$ keinen Einfluss auf die Beobachtbarkeit hat. Für diesen Fall liefert das Maß nach Tarokh ein widersprüchliches Ergebnis, da es mit verschwindendem $\alpha$ gegen Null strebt.

\subsection{Modifiziertes Modalmaß}

Nachfolgend wird eine Modifikation des Maßes nach Litz vorgeschlagen, die das gezeigte Problem behebt. Dazu wird bei gegebenem Eigenwert $\lambda$ die Singulärwertzerlegung

$$
\lambda \mathbf{E}-\mathbf{A}=\mathbf{U} \boldsymbol{\Sigma} \mathbf{V}^{\mathrm{H}}=\mathbf{U} \operatorname{diag}\left(\sigma_{j}\right) \mathbf{V}^{\mathrm{H}}
$$

der Matrix $\lambda \mathbf{E}-\mathbf{A}$ betrachtet. Die in der Diagonalmatrix $\boldsymbol{\Sigma}$ enthaltenen (geordneten) Singulärwerte werden dabei mit $\sigma_{1} \geq \sigma_{2} \geq \ldots \geq \sigma_{n}=0$ bezeichnet. Die $n \times n$ Matrizen $\mathbf{U}$ und $\mathbf{V}$ sind unitär.

Für die weiteren Betrachtungen werden die Spalten der Matrix V

$$
\mathbf{V}=:\left[\begin{array}{lll}
\mathbf{v}_{1} & \ldots & \mathbf{v}_{n}
\end{array}\right],
$$

benutzt. Insbesondere werden die Matrizen

$$
\mathbf{P}_{i}:=\left[\begin{array}{llll}
\mathbf{v}_{i} & \mathbf{v}_{i+1} & \ldots & \mathbf{v}_{n}
\end{array}\right],
$$

für $i=1, \ldots, n$ betrachtet, die sich aus den letzten $n-i+1$ Spalten von $\mathbf{V}$ ergeben. Da (zumindest) der letzte Singulärwert $\sigma_{n}$ gleich Null ist, ist $\mathbf{v}_{n}(\mathrm{~d}$. h. die einzige Spalte der Matrix $\mathbf{P}_{n}$ ) ein Eigenvektor zum betrachteten Eigenwert $\lambda$. Mitunter gilt dies aber auch für weitere Spalten von $\mathbf{V}$; ist $\lambda$ ein mehrfacher Eigenwert mit geometrischer Vielfachheit $q$, so gilt $\sigma_{n-q+1}=\ldots=\sigma_{n-1}=\sigma_{n}=0$ und die Spalten von $\mathbf{P}_{n-q+1}$ spannen den $q$-dimensionalen Eigenraum zu $\lambda$ auf. Ist in diesem Fall die Matrix $\mathbf{C P}_{n-q+1}$ nicht spaltenregulär, gilt also $\mathbf{C p}=\mathbf{0}$ für eine Linearkombination $\mathbf{p}$ der Spalten von $\mathbf{P}_{n-q+1}$, so ist das System nicht beobachtbar. In einem solchen Fall gilt nämlich

$$
\mathbf{H}_{\mathrm{b}}(\lambda) \cdot \mathbf{p}=\mathbf{0},
$$

d. h. die Matrix $\mathbf{H}_{\mathrm{b}}$ weist einen Rangverlust auf.

Dadurch motiviert werden zur Beurteilung der Spaltenregulärität der Matrizen $\mathbf{C P}_{i}(i=1, \ldots, n)$ die Zahlen

$$
\gamma_{i}:=\sqrt{\sigma_{\min }\left(\mathbf{P}_{i}^{\mathrm{H}} \mathbf{C}^{\mathrm{T}} \mathbf{C P}_{i}\right)}= \begin{cases}\sigma_{\min }\left(\mathbf{C P}_{i}\right) & \text { rang } \mathbf{P}_{i} \leq m \\ 0 & \text { sonst }\end{cases}
$$

eingeführt, wobei $\sigma_{\min }$ jeweils den kleinsten Singulärwert der Matrix bezeichnet. Diese Zahlen $\gamma_{i}$ weisen ein zu den Singulärwerten $\sigma_{1}, \ldots, \sigma_{n}$ gegenläufiges Verhalten auf. Erstere werden mit zunehmendem Index größer, während letztere kleiner werden:

$$
\begin{gathered}
\sigma_{1} \geq \sigma_{2} \geq \ldots \geq \sigma_{n}=0 \\
\sqrt{\sigma_{\min }\left(\mathbf{C}^{\mathrm{T}} \mathbf{C}\right)}=\gamma_{1} \leq \gamma_{2} \leq \ldots \leq \gamma_{n}=\sqrt{\mathbf{v}_{n}^{\mathrm{H}} \mathbf{C}^{\mathrm{T}} \mathbf{C} \mathbf{v}_{n}} .
\end{gathered}
$$

Anhand der vorangegangenen Überlegungen ist klar, dass der Eigenwert $\lambda$ genau dann nicht beobachtbar ist, d.h. rang $\mathbf{H}_{\mathrm{b}}(\lambda)<n$, wenn es einen Index $l$ gibt, für den $\sigma_{l}=\gamma_{l}=0$ gilt. Es wird daher

$$
\eta_{\lambda}(\mathbf{A}, \mathbf{C}):=\min _{l} \sqrt{\sigma_{l}^{2}+\gamma_{l}^{2}}
$$

als modifizierte Maßzahl zur Bewertung der Beobachtbarkeit des Eigenwertes $\lambda$ vorgeschlagen ( $\sigma_{l}$ und $\gamma_{l}$ hängen hier klarerweise von A, $\mathbf{C}$ und $\lambda \mathrm{ab}$ ). Anhand der Ungleichung

$$
\eta_{\lambda}(\mathbf{A}, \mathbf{C}) \leq \sqrt{\sigma_{n}^{2}+\gamma_{n}^{2}}=\gamma_{n} \leq\|\mathbf{C}\|_{2}
$$

ist offensichtlich, dass diese Maßzahl nach oben jedenfalls durch die Spektralnorm $\|\mathbf{C}\|_{2}$ der Matrix $\mathbf{C}$ beschränkt ist. Dieser Umstand erlaubt es, den erhaltenen Zahlenwert $\eta$ auf einer Skala zwischen nicht beobachtbarem $(\eta=0)$ und perfekt beobachtbarem System $\left(\eta=\|\mathbf{C}\|_{2}\right)$ einzuordnen; letzterer Fall liegt z. B. für $\mathbf{C}=\mathbf{E}, \mathrm{d}$. h. bei Messung aller Zustandsgrößen vor.

Unter der Voraussetzung einfacher Eigenwerte ist $\gamma_{n}$ gleich der Wurzel des ursprünglichen Maßes $\sqrt{\kappa_{\lambda}}$. Man erkennt dies anhand von (18b) unter Berücksichtigung der Tatsache, dass $\mathbf{v}_{n}$ ein Eigenvektor zum Eigenwert $\lambda$ mit $\mathbf{v}_{n}^{\mathrm{H}} \mathbf{v}_{n}=1$ ist. Damit hängt das modifizierte Maß $\eta_{\lambda}$ mit der ursprünglichen Maßzahl $\kappa_{\lambda}$ über die Ungleichung ${ }^{2}$

$$
\eta_{\lambda} \leq \sqrt{\kappa_{\lambda}}
$$

zusammen, wobei Gleichheit gilt, wenn das Minimum in (19) für $l=n$ angenommen wird.

2 Die hier auftretende Wurzel ist in Relation (19) bedingt; deren Einführung hat den kosmetischen Grund, dass eine Verdopplung der Ausgangsmatrix C so zu einer Verdopplung des Maßes führt, anstatt wie beim ursprünglichen Maß zu einer Vervierfachung. 


\subsection{Beispiel}

Es wird erneut das System mit den in (10) angegebenen Daten betrachtet. Für den Eigenwert $s_{1}=1$ erhält man für $\mathrm{C}=\mathrm{C}_{1}$ bzw. $\mathrm{C}=\mathrm{C}_{2}$

$$
\begin{array}{ll}
\sigma_{1}=|\alpha|, & \sigma_{2}=0 ; \\
\gamma_{1}=\left\{\begin{array}{lll}
0 & \mathbf{C}=\mathbf{C}_{1} & \gamma_{2}=1 . \\
1 & \mathbf{C}=\mathbf{C}_{2}, &
\end{array}\right.
\end{array}
$$

Gleiches gilt für $s_{2}=1+\alpha$. Die Maßzahlen ergeben sich zu

$$
\eta_{s_{1}}=\eta_{s_{2}}= \begin{cases}\min (|\alpha|, 1)<\sqrt{2}=\|\mathbf{C}\|_{2} & \mathbf{C}=\mathbf{C}_{1} \\ \min \left(\sqrt{1+\alpha^{2}}, 1\right)=1=\|\mathbf{C}\|_{2} & \mathbf{C}=\mathbf{C}_{2} .\end{cases}
$$

Vergleicht man diese mit der Wurzel der ursprünglichen Maßzahlen nach Litz

$$
\sqrt{\kappa_{s_{1}}}=\sqrt{\kappa_{s_{2}}}=1,
$$

so erkennt man, dass für $\mathbf{C}=\mathbf{C}_{1}$ bei geringem Abstand der Eigenwerte deren Differenz $\alpha$ automatisch in das Maß eingeht und so den Beobachtbarkeitsverlust bei einem zweifachen Eigenwert anzeigt. Für $\mathbf{C}=\mathbf{C}_{2}$ dagegen zeigt das Maß durch Gleichheit mit der oberen Schranke (20) die vollständige Messbarkeit aller Zustandsgrößen an.

\subsection{Eigenschaften des modifizierten Maßes}

Neben der Eigenschaft der Konsistenz, d. h.

$$
\eta_{\lambda}=0 \Leftrightarrow \quad \operatorname{rang} \mathbf{H}_{\mathrm{b}}(\lambda)<n,
$$

die im Rahmen der Konstruktion des modifizierten Maßes gezeigt wurde, ist das vorgeschlagene Maß invariant gegenüber unitären Zustandstransformationen und stetig in den Systemdaten. Die Invarianz gegenüber unitären $\mathrm{Zu}$ standstransformationen ist dabei eine direkte Konsequenz der Invarianz der Singulärwerte bezüglich der Multiplikation mit unitären Matrizen und lässt sich leicht überprüfen. Die Stetigkeit ist nicht unmittelbar ersichtlich. Hier ist zunächst lediglich klar, dass die Eigenwerte der Matrix A stetig in deren Einträgen sind, und somit auch die (nachfolgend mit $\mathbf{M}$ abgekürzte) Matrix $\lambda \mathbf{E}-\mathbf{A}$ stetig in $\mathbf{A}$ ist. Die in $\mathbf{V}$ enthaltenen Singulärvektoren sind jedoch nicht notwendigerweise stetig in $\mathbf{M}$. Insbesondere ist $\mathbf{V}$ nicht einmal eindeutig festgelegt, z. B. sind

$$
\mathbf{M}=\mathbf{U} \boldsymbol{\Sigma} \mathbf{V}^{\mathrm{H}}=(-\mathbf{U}) \boldsymbol{\Sigma}(-\mathbf{V})^{\mathrm{H}}
$$

zwei mögliche Singulärwertzerlegungen von M. In [2] wird die Existenz und Berechnung einer stetigen Singulärwert- zerlegung gezeigt; dies erfolgt jedoch unter der Voraussetzung paarweise verschiedener Singulärwerte, wovon hier nicht ausgegangen werden kann.

Zum Nachweis der Stetigkeit wird daher folgendes Lemma formuliert, welches den Einfluss von Änderungen der Matrizen $\mathbf{M}$ und $\mathbf{C}$ auf die Zahlen $\sigma_{j}$ und $\gamma_{j}$ angibt. Sein Beweis basiert auf Ergebnissen der MatrizenStörtheorie $[1,22,24,25]$ und ist im Anhang angegeben.

Lemma 1. Den Matrizen $\mathbf{M}:=\lambda \mathbf{E}-\mathbf{A}$ und $\mathbf{C}$ bzw. $\tilde{\mathbf{M}}:=\tilde{\lambda} \mathbf{E}-\tilde{\mathbf{A}}$ und $\tilde{\mathbf{C}}$ seien auf die in Abschnitt 2.2 beschriebene Weise die Zahlen $\sigma_{j}$ und $\gamma_{j} b z w . \tilde{\sigma}_{j}$ und $\tilde{\gamma}_{j}$ $(j=1, \ldots, n)$ zugeordnet ${ }^{3}$. Weiterhin seien für nichtnegative Zahlen $\varepsilon$ und $\delta$ die Ungleichungen

$$
\|\mathbf{M}-\tilde{\mathbf{M}}\|_{2} \leq \varepsilon, \quad\|\mathbf{C}-\tilde{\mathbf{C}}\|_{2} \leq \delta
$$

erfüllt. Dann gilt

$$
\begin{array}{rlrl}
\left|\sigma_{r}-\tilde{\sigma}_{r}\right| \leq \varepsilon & & \text { für } r=1, \ldots, n \\
\left|\gamma_{r}-\tilde{\gamma}_{r}\right| & =0 & & \text { für } r=1, \ldots, n-m .
\end{array}
$$

Für $r=n-m+1, \ldots, n$ gilt mit der formalen Festlegung $\sigma_{0}:=\infty$

$$
\left|\gamma_{r}-\tilde{\gamma}_{r}\right| \leq \delta+\frac{3 \varepsilon}{\sigma_{r-1}-\sigma_{r}}\|\mathbf{C}\|_{2}
$$

jeweils unter der Voraussetzung, dass $\varepsilon$ die Ungleichung

$$
0 \leq \frac{3 \varepsilon}{\sigma_{r-1}-\sigma_{r}} \leq 1
$$

erfüllt.

Gemäß diesem Lemma hängt für festen Index $r$ die Zahl $\sigma_{r}$ immer und die Zahl $\gamma_{r}$ unter der Voraussetzung $\sigma_{r-1}>\sigma_{r}$ (oder $r=1$ ) stetig von $\mathbf{A}$ und $\mathbf{C}$ ab. Unstetigkeitsstellen können also höchstens bei $\gamma_{r}$ auftreten, wenn $\sigma_{r-1}=\sigma_{r}$ gilt. Diese können aber keine Unstetigkeit des Maßes selbst zur Folge haben. In diesem Fall wird nämlich wegen der aus $\gamma_{r-1} \leq \gamma_{r}$ resultierenden Ungleichung

$$
\sqrt{\sigma_{r-1}^{2}+\gamma_{r-1}^{2}}=\sqrt{\sigma_{r}^{2}+\gamma_{r-1}^{2}} \leq \sqrt{\sigma_{r}^{2}+\gamma_{r}^{2}}
$$

das Minimum in (19) niemals für $l=r$ angenommen, sodass $\gamma_{r}$ nicht in das Maß eingeht.

3 Für die Gültigkeit des Lemmas ist es nicht erforderllich, dass $\lambda$ bzw. $\tilde{\lambda}$ Eigenwerte der Matrizen A bzw. Ã sind. 


\subsection{Beispiel zum Einfluss fehlerhafter Systemdaten}

Oft sind die Daten eines Systems nicht exakt bekannt. In solch einem Fall ist es von Interesse, wie stark sich das Beobachtbarkeitsmaß bei kleinen Änderungen der Matrizen $\mathbf{A}$ und $\mathbf{C}$ ändern kann. Anhand eines Beispiels wird demonstriert, dass Lemma 1 auch zur Beantwortung dieser Frage herangezogen werden kann.

Es wird das System aus Relation (10) mit $\mathbf{C}=\mathbf{C}_{1}$ betrachtet. Die realen Systemdaten seien nun durch die Matrizen $\tilde{\mathbf{A}}$ und $\tilde{\mathbf{C}}$ mit

$$
\|\mathbf{A}-\tilde{\mathbf{A}}\|_{2} \leq \frac{\varepsilon}{2} \quad \mathbf{C}=\tilde{\mathbf{C}}
$$

gegeben. Es wird die Änderung des zugehörigen Maßes $\tilde{\eta}_{\tilde{s}_{1}}$ gegenüber dem zuvor für den Eigenwert $s_{1}=1$ erhaltenen Maß

$$
\eta_{s_{1}}=\min (|\alpha|, 1)
$$

untersucht.

Da die Systemmatrix A Diagonalstruktur aufweist, kann die Differenz der Eigenwerte $s_{1}$ und $\tilde{s}_{1}$ der Matrizen A und $\tilde{\mathbf{A}}$ durch

$$
\left|s_{1}-\tilde{s}_{1}\right| \leq\|\mathbf{A}-\tilde{\mathbf{A}}\|_{2} \leq \frac{\varepsilon}{2}
$$

abgeschätzt werden [22]. Unter Verwendung der Nomenklatur von Lemma 1 gilt also

$$
\begin{aligned}
\|\mathbf{M}-\tilde{\mathbf{M}}\|_{2} & =\left\|\left(s_{1} \mathbf{E}-\mathbf{A}\right)-\left(\tilde{s}_{1} \mathbf{E}-\tilde{\mathbf{A}}\right)\right\|_{2} \\
& \leq\left|s_{1}-\tilde{s}_{1}\right|\|\mathbf{E}\|_{2}+\|\mathbf{A}-\tilde{\mathbf{A}}\|_{2} \leq \varepsilon .
\end{aligned}
$$

Unabhängig von Ã gilt prinzipiell $\tilde{\sigma}_{2}=\tilde{\gamma}_{1}=0$, d. h.

$$
\tilde{\eta}_{\tilde{s}_{1}}=\min \left(\tilde{\sigma}_{1}, \tilde{\gamma}_{2}\right) .
$$

Es werden nun die folgenden drei Fälle unterschieden:

$$
|\alpha|>3 \sqrt{2} \varepsilon, \quad 3 \sqrt{2} \varepsilon \geq|\alpha|>3 \varepsilon, \quad 3 \varepsilon \geq|\alpha| .
$$

Gilt $|\alpha|>3 \sqrt{2} \varepsilon$, so erhält man anhand von Lemma 1 die Abschätzungen

$$
\begin{aligned}
& \tilde{\sigma}_{1} \in[|\alpha|-\varepsilon, \quad|\alpha|+\varepsilon], \\
& \tilde{\gamma}_{2} \in\left[\begin{array}{ll}
1-\frac{3 \sqrt{2}}{|\alpha|} \varepsilon, & \left.1+\frac{3 \sqrt{2}}{|\alpha|} \varepsilon\right] .
\end{array}\right.
\end{aligned}
$$

In diesem Fall gilt also

$$
\begin{aligned}
& \tilde{\eta}_{\tilde{s}_{1}} \geq \min \left(|\alpha|-\varepsilon, \quad 1-\frac{3 \sqrt{2}}{|\alpha|} \varepsilon\right)>0, \\
& \tilde{\eta}_{\tilde{s}_{1}} \leq \min \left(|\alpha|+\varepsilon, \quad 1+\frac{3 \sqrt{2}}{|\alpha|} \varepsilon\right) .
\end{aligned}
$$

Für $3 \sqrt{2} \varepsilon \geq|\alpha|>3 \varepsilon$ gelten die Abschätzungen

$$
\begin{aligned}
& \tilde{\sigma}_{1} \in\left[\begin{array}{ll}
|\alpha|-\varepsilon, & |\alpha|+\varepsilon
\end{array}\right], \\
& \tilde{\gamma}_{2} \in\left[\begin{array}{ll}
0, & 1+\frac{3 \sqrt{2}}{|\alpha|} \varepsilon
\end{array}\right]
\end{aligned}
$$

und man erhält

$$
0 \leq \tilde{\eta}_{\tilde{s}_{1}} \leq \min \left(|\alpha|+\varepsilon, \quad 1+\frac{3 \sqrt{2}}{|\alpha|} \varepsilon\right) .
$$

Gilt schließlich $3 \varepsilon \geq|\alpha|$, so ergeben sich unter Berücksichtigung von $\tilde{\gamma}_{2} \leq\|\mathbf{C}\|_{2}$ die Abschätzungen

$$
\begin{aligned}
& \tilde{\sigma}_{1} \in[\max (0,|\alpha|-\varepsilon), \quad|\alpha|+\varepsilon], \\
& \tilde{\gamma}_{2} \in\left[\begin{array}{ll}
0, & \sqrt{2}
\end{array}\right]
\end{aligned}
$$

und damit

$$
0 \leq \tilde{\eta}_{\tilde{s}_{1}} \leq|\alpha|+\varepsilon \leq 4 \varepsilon .
$$

In Abbildung 1 sind das ursprüngliche Maß $\kappa_{s_{1}}$, das modifizierte Maß $\eta_{s_{1}}$ sowie die ermittelten oberen und unteren Schranken von $\tilde{\eta}_{\tilde{s}_{1}}$ für zwei Werte von $\varepsilon$ dargestellt. Man kann erkennen bzw. auch anhand der vorangegangenen Rechnung verifizieren, dass beispielsweise für $|\alpha|>0,5$ das Maß $\tilde{\eta}_{\tilde{s}_{1}}$ (für $\varepsilon=0,1$ ) garantiert ungleich Null ist. Daraus kann trotz ungenauer Kenntnis der Systemdaten $\tilde{\mathbf{A}}, \tilde{\mathbf{C}}$ auf die Beobachtbarkeit des Systems geschlossen werden.

….. Ursprüngliches Maß $\kappa_{s_{1}}$
- Modifiziertes Maß $\eta_{s_{1}}$
- - - obere Schranke von $\tilde{\eta}_{\tilde{s}_{1}}$ für $\varepsilon=0,1$
---- obere Schranke von $\tilde{\eta}_{\tilde{s}_{1}}$ für $\varepsilon=0,05$
- - - untere Schranke von $\tilde{\eta}_{\tilde{s}_{1}}$ für $\varepsilon=0,1$
---- untere Schranke von $\tilde{\eta}_{\tilde{s}_{1}}$ für $\varepsilon=0,05$

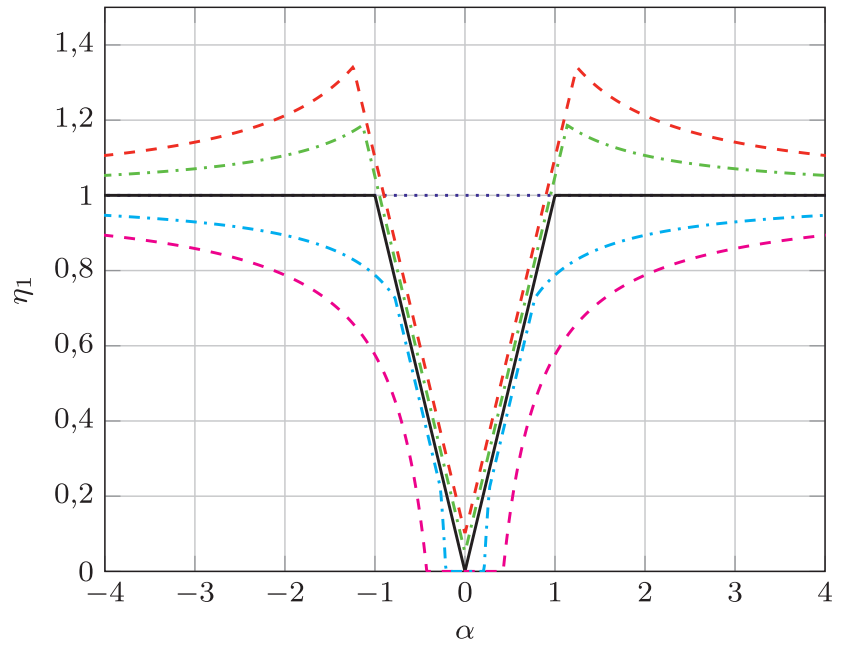

Abbildung 1: Ursprüngliches Modalmaß $\kappa_{s_{1}}$, modifiziertes Modalmaß $\eta_{s_{1}}$ sowie aus $\|\mathbf{A}-\tilde{\mathbf{A}}\|_{2} \leq \frac{\varepsilon}{2}$ resultierende Schranken von $\tilde{\eta}_{\tilde{s}_{1}}$ für zwei Werte von $\varepsilon$. 


\section{Perspektivische Beobachtbarkeit}

Wie in der Einleitung angemerkt, ist die perspektivische Beobachtbarkeit u. a. in der Bildverarbeitung von Bedeutung. Dies wird zunächst anhand eines anschaulichen Beispiels aus diesem Bereich diskutiert. Anschließend wird auf das erwähnte Kriterium (4) eingegangen. Davon ausgehend wird das erweiterte Modalmaß auf die perspektivische Beobachtbarkeit übertragen und anschließend auf das Beispiel angewandt.

\subsection{Einleitendes Beispiel}

Zur Veranschaulichung der Zusammenhänge wird ein Körper im dreidimensionalen Raum betrachtet, welcher einer Drehung und einer Linearbewegung unterworfen ist. Konkret erfüllt ein Punkt p auf diesem Körper die Differentialgleichung

$$
\frac{\mathrm{dp}}{\mathrm{d} t}=\mathbf{\Omega} \mathbf{p}+\mathbf{v}
$$

Dabei ist $\mathbf{v}$ ein konstanter Geschwindigkeitsvektor und die schiefsymmetrische Matrix $\Omega$ ergibt sich aus dem Parametervektor $\boldsymbol{\omega}:=\left[\begin{array}{lll}\omega_{1} & \omega_{2} & \omega_{3}\end{array}\right]^{\mathrm{T}}$ gemäß

$$
\boldsymbol{\Omega}:=\left[\begin{array}{ccc}
0 & -\omega_{3} & \omega_{2} \\
\omega_{3} & 0 & -\omega_{1} \\
-\omega_{2} & \omega_{1} & 0
\end{array}\right]
$$

Wie man leicht ermitteln kann, lautet das charakteristische Polynom dieser Matrix

$$
\operatorname{det}(s \mathbf{E}-\boldsymbol{\Omega})=s\left(s^{2}+\boldsymbol{\omega}^{\mathrm{T}} \boldsymbol{\omega}\right)
$$

Die Winkelgeschwindigkeit der Drehung ist dabei durch $\omega:=\sqrt{\boldsymbol{\omega}^{\mathrm{T}} \boldsymbol{\omega}}$ gegeben. Der Vektor $\boldsymbol{\omega}$ ist Eigenvektor zum Eigenwert Null und zeigt in Richtung der Rotationsachse.

Dieser Körper bzw. insbesondere der Punkt p werde durch eine Kamera gefilmt. Damit wird auf deren Bildebene die perspektivische Projektion $\tilde{\mathbf{p}}$ des Punktes $\mathbf{p}$ abgebildet. Ist der Brennpunkt der Kamera durch $\mathbf{f}$ gegeben und die Bildebene der Kamera durch die Gleichung $\mathbf{f}^{\mathrm{T}} \tilde{\mathbf{p}}=0$ bestimmt, so lässt sich dieser Zusammenhang durch

$$
\tilde{\mathbf{p}}=\frac{\mathbf{E}-\frac{\mathbf{f} \cdot \mathbf{f}^{\mathrm{T}}}{\mathbf{f}^{\mathrm{T}} \cdot \mathbf{f}}}{1-\frac{\mathbf{f}^{\mathrm{T}} \cdot \mathbf{p}}{\mathbf{f}^{\mathrm{T}} \cdot \mathbf{f}}} \mathbf{p}
$$

mathematisch beschreiben. Die Brennweite der Kamera hat dabei den Wert $f:=\sqrt{\mathbf{f}^{\mathrm{T}} \mathbf{f}}$.
In Abbildung 2 sind beispielhafte Trajektorien des Punktes p und des zugehörigen Bildpunktes $\tilde{\mathbf{p}}$ für die Parameterwerte

$$
\begin{aligned}
& \boldsymbol{\omega}=\left[\begin{array}{lll}
-1 & 2 & 2
\end{array}\right]^{\mathrm{T}}, \\
& \mathbf{v}=\left[\begin{array}{lll}
1 & 0 & 0
\end{array}\right]^{\mathrm{T}}, \\
& \mathbf{f}=\left[\begin{array}{lll}
0 & -1 & 0
\end{array}\right]^{\mathrm{T}}
\end{aligned}
$$

dargestellt. Bildebene ist in diesem Fall die durch $y=0$ charakterisierte $x$-z-Ebene, die Brennweite beträgt $f=1$.

Führt man den Zustandsvektor $\mathbf{x}:=\left[\begin{array}{ll}w \mathbf{p}^{\mathrm{T}} & w\end{array}\right]^{\mathrm{T}}$ mit der konstanten Größe $w \neq 0$ ein, so ist $\mathbf{p}$ eine mögliche Darstellung von $\mathbf{x}$ in homogenen Koordinaten, d. h. $[\mathbf{p}]=[\mathbf{x}]$. Durch Festlegung der Ausgangsgröße

$$
\mathbf{y}:=\beta\left[\begin{array}{c}
w\left(\mathbf{E}-\frac{\mathbf{f} \cdot \mathbf{f}^{\mathrm{T}}}{\mathbf{f}^{\mathrm{T}} \cdot \mathbf{f}}\right) \mathbf{p} \\
w\left(1-\frac{\mathbf{f}^{\mathrm{T}} \cdot \mathbf{p}}{\mathbf{f}^{\mathrm{T}} \cdot \mathbf{f}}\right)
\end{array}\right]
$$

mit der (beliebigen) Konstante $\beta \neq 0$ besteht derselbe $\mathrm{Zu}$ sammenhang zwischen $\tilde{\mathbf{p}}$ und $\mathbf{y}$. Man erhält somit ein System der Form (1) mit den Daten

$$
\begin{aligned}
& \mathbf{A}=\left[\begin{array}{ll}
\boldsymbol{\Omega} & \mathbf{v} \\
\mathbf{0}^{\mathrm{T}} & 0
\end{array}\right]=\left[\begin{array}{cccc}
0 & -2 & 2 & 1 \\
2 & 0 & 1 & 0 \\
-2 & -1 & 0 & 0 \\
0 & 0 & 0 & 0
\end{array}\right], \\
& \mathbf{C}=\beta\left[\begin{array}{cc}
\mathbf{E}-\frac{\mathbf{f}^{\mathrm{T}}}{\mathbf{f}^{\mathrm{T}} \mathbf{f}} & \mathbf{0} \\
-\frac{\mathbf{f}^{\mathrm{T}}}{\mathbf{f}^{\mathrm{T}} \mathbf{f}} & 1
\end{array}\right]=\left[\begin{array}{llll}
\beta & 0 & 0 & 0 \\
0 & 0 & 0 & 0 \\
0 & 0 & \beta & 0 \\
0 & \beta & 0 & \beta
\end{array}\right],
\end{aligned}
$$

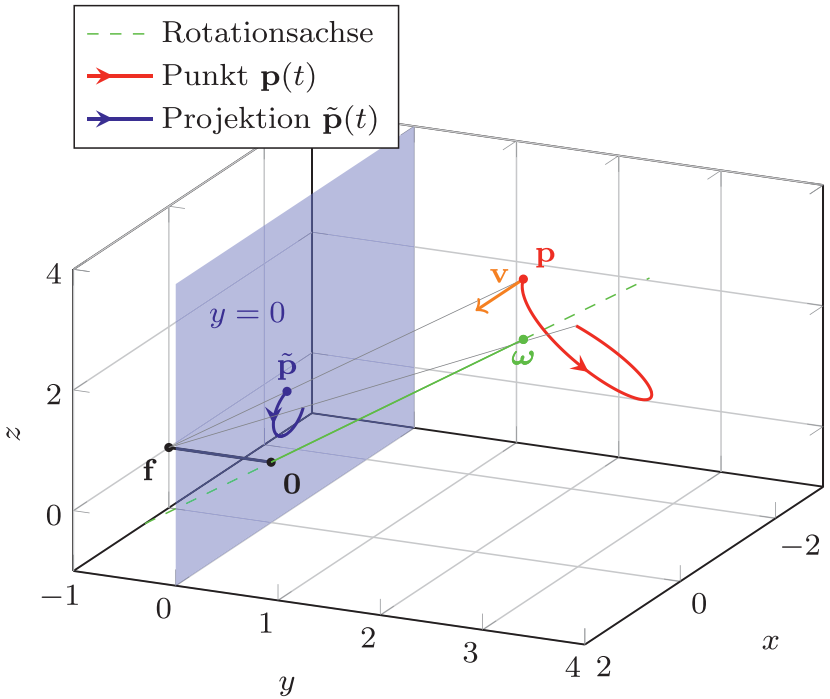

Abbildung 2: Trajektorien des Punktes p und dessen perspektivischer Projektion $\tilde{\mathbf{p}}$ in die $x$ - $z$-Ebene. 
wobei sich die angegebenen Zahlenwerte für die Parameter aus Relation (47) ergeben. Man erkennt, dass die zweite Komponente von y bzw. die zweite Zeile von $\mathbf{C}$ gleich Null sind und daher entfernt werden könnten. Ihre Beibehaltung hat jedoch keinen Einfluss auf die Ergebnisse. Für die Rekonstruierbarkeit des Punktes $\mathbf{p}$ bei Kenntnis einer Trajektorie des Bildpunktes $\tilde{\mathbf{p}}$ ist damit die perspektivische Beobachtbarkeit dieses Systems (für einen beliebigen, konkreten Wert von $\beta$ ) ausschlaggebend.

\subsection{Kriterium}

Bevor das in [3] angegebene Kriterium für die perspektivische Beobachtbarkeit formuliert wird, müssen zwei Fälle unterschieden werden. Ist der Anfangszustand des Systems auf reelle Werte eingeschränkt, gilt also $\mathbf{x}_{0} \in \mathbb{R}^{n}$, so spricht man von perspektivischer Beobachtbarkeit über $\mathbb{R}$, andernfalls - wenn $\mathbf{x}_{0}$ komplexwertig sein kann - von perspektivischer Beobachtbarkeit über $\mathbb{C}$. Nur über $\mathbb{C}$ existiert ein (uneingeschränkt gültiges ${ }^{4}$ ) notwendiges und hinreichendes Kriterium:

Satz 1 ([3]). Das System (1) ist genau dann perspektivisch beobachtbar über $\mathbb{C}$, wenn die in Relation (4) definierte Matrix $\mathbf{H}_{\mathrm{p}}$ für alle Paare $\lambda_{1}, \lambda_{2}$ je zweier (nicht notwendigerweise unterschiedlicher) Eigenwerte der Matrix A die Rangbedingung

$$
\operatorname{rang} \mathbf{H}_{\mathrm{p}}\left(\lambda_{1}, \lambda_{2}\right)=n
$$

erfüllt. Über $\mathbb{R}$ ist diese Bedingung nur hinreichend, nicht aber notwendig.

Mit der Abkürzung $\Lambda(\mathbf{A})$ für die Menge der Eigenwerte von A gelten somit die Zusammenhänge

Rangbedingung (50)
$\forall \lambda_{1}, \lambda_{2} \in \Lambda(\mathbf{A})$$\Leftrightarrow \begin{aligned} & \text { perspektivisch } \\ & \text { beob. über } \mathbb{C}\end{aligned} \Rightarrow \begin{aligned} & \text { perspektivisch } \\ & \text { beob. über } \mathbb{R} .\end{aligned}$

Für die folgenden Betrachtungen zur Bewertung der perspektivischen Beobachtbarkeit wird ausschließlich der komplexwertige Fall betrachtet, für welchen das Rangkriterium notwendig und hinreichend ist. Dies mag widersprüchlich erscheinen, da auf diese Weise nur eine Bewertung der perspektivischen Beobachtbarkeit über $\mathbb{C}$ möglich ist, während beim diskutierten Beispiel offensichtlich der reellwertige Fall von praktischer Bedeutung ist. Als Motivation dient dabei, dass ein über $\mathbb{C}$ nicht (oder

4 Kriterien für die perspektivische Beobachtbarkeit über $\mathbb{R}$, die unter zusätzlichen Voraussetzungen bezüglich der Systemmatrix A notwendig und hinreichend sind, werden in $[4,19]$ untersucht. „schlecht“) perspektivisch beobachtbares System, auch wenn es über $\mathbb{R}$ („gut“) perspektivisch beobachtbar ist, aus praktischer Sicht ein Problem darstellt: Es bedarf nämlich auf jeden Fall einer weiteren Untersuchung, die mangels dafür geeigneter Kriterien mitunter nicht ohne weiteres durchführbar ist.

Durch Einführen des sogenannten Dimensionsverlustes $d$ ist es möglich, Definition 1 sowie Satz 1 zu verallgemeinern [7]; dabei erhält man für $d=0$ die klassische Beobachtbarkeit und für $d=1$ den diskutierten Fall perspektivischer Beobachtbarkeit. Diese Verallgemeinerung wird der Einfachheit halber hier nicht betrachtet, die nachfolgenden Überlegungen lassen sich aber geradlinig darauf übertragen.

\subsection{Modalmaß für perspektivische Beobachtbarkeit}

Die Erweiterung von Definition 2 zu einer Definition von Maßen für die perspektivische Beobachtbarkeit ist evident. Für Modalmaße wird in diesem Zusammenhang folgende Definition benutzt:

Definition 4. Eine Funktion $v_{\lambda}(\mathrm{A}, \mathrm{C})$ heißt modales Maß für die perspektivische Beobachtbarkeit, wenn sie für Eigenwerte $\lambda$ von $\mathbf{A}$, d. h. für $\operatorname{det}(\lambda \mathbf{E}-\mathbf{A})=0$, folgende Bedingungen erfüllt:

1. Nichtnegativität: $v_{\lambda}(\mathbf{A}, \mathbf{C}) \geq 0$.

2. Konsistenz: $\quad v_{\lambda}(\mathbf{A}, \mathbf{C})=0 \Leftrightarrow$ für ein $\lambda_{2}$ gilt rang $\mathbf{H}_{\mathrm{p}}\left(\lambda, \lambda_{2}\right)<n$, d. h. ein $\lambda$ enthaltendes Eigenwertpaar ist nicht perspektivisch beobachtbar.

3. Stetigkeit: $v_{\lambda}(\mathrm{A}, \mathrm{C})$ ist stetig in $\mathrm{A}, \mathrm{C}, \lambda$.

Das in Abschnitt 2.2 vorgestellte modifizierte Beobachtbarkeitsmaß lässt sich in relativ einfacher Weise auf die perspektivische Beobachtbarkeit über $\mathbb{C}$ erweitern: Zur Bewertung der perspektivischen Beobachtbarkeit des Eigenwertpaars $\left(\lambda_{1}, \lambda_{2}\right)$ reicht es aus, die Matrix $\lambda \mathbf{E}-\mathbf{A}$ in Relation (13) durch

$$
\left(\lambda_{1} \mathbf{E}-\mathbf{A}\right)\left(\lambda_{2} \mathbf{E}-\mathbf{A}\right)=\mathbf{U} \operatorname{diag}\left(\sigma_{j}\right) \mathbf{V}^{\mathrm{H}}
$$

zu ersetzen. Die zum Singulärwert Null gehörigen Spalten von $\mathbf{V}$ stellen damit Linearkombinationen von Rechtseigenvektoren (bzw. bei doppelten Eigenwerten ggf. von Eigen- und Hauptvektoren) zu diesen Eigenwerten dar.

Für die perspektivische Beobachtbarkeit über $\mathbb{C}$ ist gemäß Satz 1 die lineare Unabhängigkeit dieser Spalten nach Multiplikation mit der Matrix $\mathbf{C}$ ausschlaggebend. Durch Berechnung der Zahlen $\gamma_{1}, \ldots, \gamma_{n}$ gemäß Relati- 
on (17) lässt sich diese beurteilen, und man kann durch die Maßzahl

$$
\chi_{\lambda_{1}, \lambda_{2}}(\mathbf{A}, \mathbf{C})=\min _{l} \sqrt{\sigma_{l}^{2}+\gamma_{l}^{2}}
$$

die perspektivische Beobachtbarkeit des betrachteten Eigenwertpaares $\lambda_{1}, \lambda_{2}$ bewerten.

Ein Modalmaß für die perspektivische Beobachtbarkeit im Sinne von Definition 4 ist dann durch das Minimum der Maßzahlen aller Eigenwertpaare gegeben, die den $\mathrm{zu}$ bewertenden Eigenwert $\lambda$ enthalten. Mit den Eigenwerten $s_{1}, \ldots, s_{n}$ der Matrix $\mathbf{A}$ ist es durch

$$
\chi_{\lambda}(\mathbf{A}, \mathbf{C}):=\min _{k} \chi_{\lambda, s_{k}}(\mathbf{A}, \mathbf{C})
$$

gegeben.

Die in Abschnitt 2.4 angegebenen Eigenschaften gelten offensichtlich (sinngemäß) auch für dieses Maß. Es handelt sich also um ein konsistentes Maß, welches stetig in $\mathbf{A}$ und $\mathbf{C}$ sowie invariant bezüglich unitärer Zustandstransformationen ist. Des weiteren kann auch hier Lemma 1 verwendet werden, um den Einfluss fehlerhafter Systemdaten auf die Maßzahlen abzuschätzen.

Der praktischen Verwendbarkeit dieses Maßes steht noch im Wege, dass die Ausgangsmatrix C, wie im Beispiel in Abschnitt 3.1 gezeigt, mitunter nur bis auf ein skalares Vielfaches festgelegt ist. Um eine sinnvolle Bewertung zu ermöglichen, ist in einem solchen Fall daher eine Normierung der Ausgangsmatrix notwendig. Eine Möglichkeit dazu, die im Hinblick auf die zu Relation (20) analoge Schranke $\chi_{\lambda} \leq\|\mathbf{C}\|_{2}$ sinnvoll erscheint, ist die Skalierung der Matrix $\mathbf{C}$ so, dass für deren Spektralnorm gilt:

$$
\|\mathrm{C}\|_{2}=1
$$

\subsection{Beispiel zur Berechnung des Modalmaßes}

Das vorgeschlagene Maß wird nun für das in Abschnitt 3.1 beschriebene Beispiel ermittelt. Als Parameter werden

$$
\begin{aligned}
\mathbf{v}=\boldsymbol{\omega} & =\left[\begin{array}{lll}
-3 \sin \psi & 5 \cos \psi & 4 \sin \psi
\end{array}\right]^{\mathrm{T}}, \\
\mathbf{f} & =\left[\begin{array}{lll}
0 & -1 & 0
\end{array}\right]^{\mathrm{T}},
\end{aligned}
$$

mit dem Winkel $\psi \in\left[-\frac{\pi}{2}, \frac{\pi}{2}\right]$ verwendet. Diese Wahl hat zur Folge, dass unabhängig von $\psi$ die Winkelgeschwindigkeit den Wert $\sqrt{\boldsymbol{\omega}^{\mathrm{T}} \boldsymbol{\omega}}=5$ hat und der Punkt sich in Richtung der Rotationsachse bewegt. Unter Berücksichtigung der Nor- mierungsbedingung (54) erhält man $\beta=\frac{1}{\sqrt{2}}$. Die Systemdaten gemäß (49a) sind damit durch

$$
\begin{aligned}
& \mathbf{A}=\left[\begin{array}{cccc}
0 & -4 \sin \psi & 5 \cos \psi & -3 \sin \psi \\
4 \sin \psi & 0 & 3 \sin \psi & 5 \cos \psi \\
-5 \cos \psi & -3 \sin \psi & 0 & 4 \sin \psi \\
0 & 0 & 0 & 0
\end{array}\right] \\
& \mathbf{C}=\frac{1}{\sqrt{2}}\left[\begin{array}{llll}
1 & 0 & 0 & 0 \\
0 & 0 & 0 & 0 \\
0 & 0 & 1 & 0 \\
0 & 1 & 0 & 1
\end{array}\right]
\end{aligned}
$$

gegeben. Die Matrix A besitzt die von $\psi$ unabhängigen Eigenwerte

$$
s_{1}=5 j, \quad s_{2}=-5 j, \quad s_{3}=0\left(=s_{4}\right),
$$

wobei der doppelte Eigenwert bei Null die geometrische Vielfachheit Eins hat.

Die Berechnung des Maßes wird nun für das Eigenwertpaar $\lambda_{1}=\lambda_{2}=s_{3}$ demonstriert. Die Singulärwerte von $\mathbf{A}^{2}$ lauten

$$
\sigma_{1}=25, \quad \sigma_{2}=25, \quad \sigma_{3}=0, \quad \sigma_{4}=0 .
$$

Somit sind nur die Zahlen $\gamma_{1}$ und $\gamma_{3}$ relevant, wobei $\gamma_{1}=0$ gilt. Die Matrix $\mathbf{P}_{3}$ enthält einen zum Eigenwert Null gehörigen Eigen- und Hauptvektor der Systemmatrix A und lautet

$$
\mathbf{P}_{3}=\left[\begin{array}{cc}
-\frac{3}{5} \sin \psi & 0 \\
\cos \psi & 0 \\
\frac{4}{5} \sin \psi & 0 \\
0 & 1
\end{array}\right] .
$$

Damit lässt sich $\gamma_{3}$ durch Berechnung der Quadratwurzel des kleinsten Eigenwertes der Matrix

$$
\mathbf{P}_{3}^{\mathrm{H}} \mathbf{C}^{\mathrm{T}} \mathbf{C P}_{3}=\frac{1}{2}\left[\begin{array}{cc}
1 & \cos \psi \\
\cos \psi & 1
\end{array}\right]
$$

bestimmen; man erhält dafür folgenden Wert, welcher aufgrund des großen Wertes von $\sigma_{1}$ zugleich der Wert der Maßzahl $\chi_{s_{3}, s_{3}}$ ist:

$$
\chi_{s_{3}, s_{3}}=\sqrt{\frac{1-|\cos \psi|}{2}} .
$$

Für die restlichen Maßzahlen erhält man auf ähnliche Weise den vom Winkel $\psi$ unabhängigen Wert

$$
\chi_{s_{1}, s_{1}}=\chi_{s_{1}, s_{2}}=\chi_{s_{1}, s_{3}}=\chi_{s_{2}, s_{2}}=\chi_{s_{2}, s_{3}}=\frac{1}{\sqrt{2}} .
$$




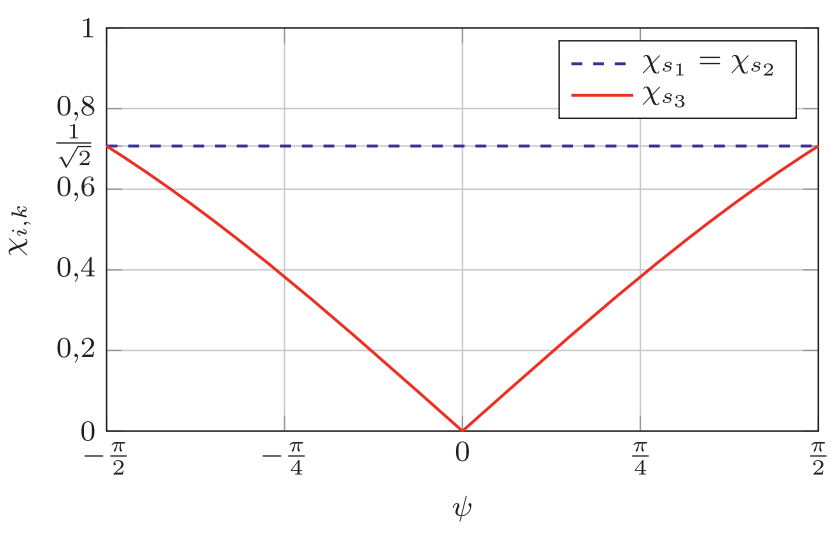

Abbildung 3: Verlauf des Modalmaßes $\chi_{s_{i}}$ für die perspektivische Beobachtbarkeit aus (63) in Abhängigkeit des Winkels $\psi$ für die Eigenwerte $s_{1}, s_{2}, s_{3}$.

Die Modalmaße der perspektivischen Beobachtbarkeit gemäß (53) lauten damit

$$
\chi_{s_{1}}=\chi_{s_{2}}=\frac{1}{\sqrt{2}}, \quad \chi_{s_{3}}=\sqrt{\frac{1-|\cos \psi|}{2}} .
$$

Abbildung 3 zeigt den Verlauf der ermittelten Maßzahlen. Man erkennt, dass es für $\psi=0 \mathrm{zu}$ einem Verlust der perspektivischen Beobachtbarkeit kommt. In diesem Fall gilt $\mathbf{v}=\boldsymbol{\omega}=-\mathbf{5 f}$, d. h. der Punkt bewegt sich in Richtung der Brennachse der Kamera und rotiert zugleich um diese. Es ist leicht vorstellbar, dass die Entfernung des Punktes von der Kamera in einem solchen Fall nicht rekonstruiert werden kann.

\section{Zusammenfassung}

Im vorliegenden Beitrag wurde auf Schwachstellen zweier existierender modaler Beobachtbarkeitsmaße eingegangen, und dadurch motiviert die Modifikation eines der Maße vorgeschlagen. Es wurde gezeigt, dass das modifizierte Maß im Gegensatz zum ursprünglichen sowohl stetig ist, als auch konsistent, d. h. bei Annäherung an ein nicht beobachtbares System strebt der Wert des Maßes gegen Null. Ein Nebenprodukt des Beweises ermöglichte dabei die Abschätzung des Einflusses kleiner Änderungen der Systemdaten auf den Wert des Maßes; dies wurde anhand eines Beispiels demonstriert. Auf die perspektivische Beobachtbarkeit, deren Bedeutung anhand eines Beispiels veranschaulicht wurde, konnte das Maß in geradliniger Weise übertragen werden. Zur praktischen Verwendung des Maßes war in diesem Fall eine Normierung der Ausgangsmatrix nötig, für welche ein Vorschlag gemacht wurde. Anhand eines Beispiels wurde schließlich die Berechnung des erweiterten Maßes demonstriert.

\section{A Beweis von Lemma 1}

Relation (28b) ist unmittelbar evident, da $\gamma_{r}=\tilde{\gamma}_{r}=0$ für $r \leq n-m$ gemäß Relation (17) gilt.

Die restlichen Behauptungen des Lemmas werden mit Methoden der Matrizen-Störtheorie [22] bewiesen. Allgemein gilt für zwei Matrizen $\mathbf{M}$ und $\tilde{\mathbf{M}}$ mit den Singulärwerten $\sigma_{j}$ und $\tilde{\sigma}_{j}$ die Ungleichung [25]

$$
\left|\sigma_{j}-\tilde{\sigma}_{j}\right| \leq\|\mathbf{M}-\tilde{\mathbf{M}}\|_{2} .
$$

Damit ist Relation (28a) eine unmittelbare Konsequenz von (27). Für $m=n$ folgt auch Relation (28c) für $r=1$, d. h.

$$
\left|\gamma_{1}-\tilde{\gamma}_{1}\right| \leq \delta,
$$

aus diesem Umstand, da in diesem Fall $\gamma_{1}=\sigma_{\min }(\mathbf{C})$ gilt.

Es verbleibt die Gültigkeit von Relation (28c) für festen Index $r \geq 2$ und $r>n-m$ zu zeigen. Dazu wird angenommen, dass $\mathbf{M}$ die Singulärwertzerlegung

$$
\mathbf{M}=\left[\begin{array}{ll}
\mathbf{U}_{1} & \mathbf{U}_{2}
\end{array}\right]\left[\begin{array}{ll}
\boldsymbol{\Sigma}_{1} & \\
& \boldsymbol{\Sigma}_{2}
\end{array}\right]\left[\begin{array}{l}
\mathbf{V}_{1}^{H} \\
\mathbf{V}_{2}^{H}
\end{array}\right]
$$

besitzt, wobei $\Sigma_{1}$ die Singulärwerte $\sigma_{1}, \ldots, \sigma_{r-1}$ und $\Sigma_{2}$ die Singulärwerte $\sigma_{r}, \ldots, \sigma_{n}$ enthält. Die in gleicher Weise partitionierte Singulärwertzerlegung von $\tilde{\mathbf{M}}$ laute

$$
\tilde{\mathbf{M}}=\left[\begin{array}{ll}
\tilde{\mathbf{U}}_{1} & \tilde{\mathbf{U}}_{2}
\end{array}\right]\left[\begin{array}{ll}
\tilde{\boldsymbol{\Sigma}}_{1} & \\
& \tilde{\boldsymbol{\Sigma}}_{2}
\end{array}\right]\left[\begin{array}{c}
\tilde{\mathbf{V}}_{1}^{H} \\
\tilde{\mathbf{V}}_{2}^{H}
\end{array}\right] .
$$

Wegen $r>n-m$ lässt sich Relation (17) in der Form

$$
\gamma_{r}=\sigma_{\min }\left(\mathbf{C V}_{2}\right), \quad \tilde{\gamma}_{r}=\sigma_{\min }\left(\tilde{\mathbf{C V}}_{2}\right)
$$

anschreiben. Die Abschätzung

$$
\left|\gamma_{r}-\tilde{\gamma}_{r}\right| \leq\left\|\mathbf{C V}_{2}-\tilde{\mathbf{C}} \tilde{\mathbf{V}}_{2}\right\|_{2}
$$

ist nun naheliegend, jedoch ist es nicht möglich, die Differenz von $\mathbf{V}_{2}$ und $\tilde{\mathbf{V}}_{2}$ in dieser Form sinnvoll abzuschätzen. Diese Matrizen sind nicht einmal eindeutig festgelegt und können sich auch bei beliebig kleinem Wert von $\varepsilon$ beträchtlich unterscheiden.

Ausschlaggebend für $\gamma_{r}$ und $\tilde{\gamma}_{r}$ sind jedoch nicht die Matrizen $\mathbf{V}_{2}$ und $\tilde{\mathbf{V}}_{2}$ selbst, sondern die von ihren Spalten aufgespannten Vektorräume. Der Abstand zwischen diesen zwei Vektorräumen der Dimension $n-r+1$ lässt sich durch die sogenannten kanonischen Winkel messen [1]. Diese stellen eine Verallgemeinerung des Winkels zwischen zwei Geraden (eindimensionalen Vektorräumen) dar. Die Anzahl $N$ dieser Winkel ist durch

$$
N=\min (n-r+1, r-1)
$$


gegeben. Im vorliegenden Fall handelt es sich um Zahlen $\varphi_{j}$ für $j=1, \ldots, N$. Diese erfüllen jeweils die Ungleichung $0 \leq \varphi_{j} \leq \frac{\pi}{2}$ und lassen sich durch Singulärwertzerlegungen der Matrizen $\tilde{\mathbf{V}}_{1}^{\mathrm{H}} \mathbf{V}_{2}$ bzw. $\tilde{\mathbf{V}}_{2}^{\mathrm{H}} \mathbf{V}_{2}$ berechnen; es gilt

$$
\begin{aligned}
\tilde{\mathbf{V}}_{1}^{\mathrm{H}} \mathbf{V}_{2} & =\mathbf{P}^{\mathrm{H}} \operatorname{diag}\left(\sin \varphi_{j}\right) \mathbf{R} \\
\tilde{\mathbf{V}}_{2}^{\mathrm{H}} \mathbf{V}_{2} & =\mathbf{Q}^{\mathrm{H}} \operatorname{diag}\left(\cos \varphi_{j}\right) \mathbf{R} .
\end{aligned}
$$

mit unitären Matrizen $\mathbf{P}, \mathbf{Q}$ und $\mathbf{R}$. Dabei wird abkürzend $\operatorname{diag}\left(\sin \varphi_{j}\right)$ bzw. $\operatorname{diag}\left(\cos \varphi_{j}\right)$ für eine (im ersten Fall nicht notwendigerweise quadratische) Matrix geschrieben, deren erste $N$ Hauptdiagonaleinträge durch $\sin \varphi_{j}$ bzw. $\cos \varphi_{j}$ und deren restliche Hauptdiagonaleinträge durch Null bzw. Eins gegeben sind; die restlichen Einträge der Matrizen sind gleich Null.

Ein Theorem von Wedin [24] liefert unter der Voraussetzung $\sigma_{r-1}-\sigma_{r}>\varepsilon$ die Abschätzung:

$$
\sin \varphi_{j} \leq\left\|\tilde{\mathbf{V}}_{1}^{\mathrm{H}} \mathbf{V}_{2}\right\|_{2} \leq \frac{\sqrt{2} \varepsilon}{\sigma_{r-1}-\sigma_{r}-\varepsilon}=: v(\varepsilon) .
$$

Deren rechte Seite wird mit $v(\varepsilon)$ abgekürzt.

Anstatt der Differenz von $\mathbf{V}_{2}$ und $\tilde{\mathbf{V}}_{2}$ betrachtet man nun die Matrix

$$
\mathbf{T}:=\mathbf{V}_{2}-\tilde{\mathbf{V}}_{2} \mathbf{Q}^{\mathrm{H}} \mathbf{R}
$$

Anhand von

$$
\begin{aligned}
\mathbf{T}^{\mathrm{H}} \mathbf{T} & =2 \mathbf{E}-\mathbf{V}_{2}^{\mathrm{H}} \tilde{\mathbf{V}}_{2} \mathbf{Q}^{\mathrm{H}} \mathbf{R}-\mathbf{R}^{\mathrm{H}} \mathbf{Q} \tilde{\mathbf{V}}_{2}^{\mathrm{H}} \mathbf{V}_{2} \\
& =2 \mathbf{R}^{\mathrm{H}} \operatorname{diag}\left(1-\cos \varphi_{j}\right) \mathbf{R}
\end{aligned}
$$

erkennt man, dass deren Singulärwerte durch $\sqrt{2-2 \cos \varphi_{j}}$ gegeben sind. Mittels der Relation $\cos ^{2} \varphi_{j}=1-\sin ^{2} \varphi_{j}$ erhält man somit

$$
\|\mathbf{T}\|_{2} \leq \sqrt{2} \cdot \sqrt{1-\sqrt{1-v^{2}(\varepsilon)}} .
$$

Man überprüft leicht, dass es sich bei der rechten Seite dieser Ungleichung um eine konvexe Funktion in $\varepsilon$ handelt: Sie ist die Verknüpfung der für $0 \leq v \leq 1$ konvexen und monoton wachsenden Funktion $\sqrt{1-\sqrt{1-v^{2}}}$ und der bei positivem Nenner konvexen Funktion $v(\varepsilon)$. An der unteren bzw. oberen Grenze des durch Relation (29) festgelegten

5 Voraussetzung für die Anwendung des Theorems ist, dass die Singulärwerte in $\tilde{\boldsymbol{\Sigma}}_{1}$ und $\boldsymbol{\Sigma}_{2}$ jeweils gänzlich in zwei disjunkten, kompakten Intervallen liegen. Die angegebene Ungleichung ergibt sich aus der Differenz zwischen dem gemäß Relation (28a) kleinstmöglichen Wert in $\tilde{\boldsymbol{\Sigma}}_{1}$ und dem größten Wert in $\boldsymbol{\Sigma}_{2}$.
Intervalls nimmt sie die Werte Null bzw. Eins an, was in diesem Bereich die Abschätzung 6

$$
\|\mathbf{T}\|_{2} \leq \frac{3 \varepsilon}{\sigma_{r-1}-\sigma_{r}}
$$

erlaubt.

Aufgrund der Unitarität der Matrix $\mathbf{Q}^{\mathrm{H}} \mathbf{R}$ gilt

$$
\tilde{\gamma}_{r}=\sigma_{\min }\left(\tilde{\mathbf{C}}_{2}\right)=\sigma_{\min }\left(\tilde{\mathbf{C}}_{\mathbf{V}_{2}} \mathbf{Q}^{\mathrm{H}} \mathbf{R}\right) .
$$

Man erhält somit folgendermaßen die behauptete Ungleichung (28c)

$$
\begin{aligned}
\left|\gamma_{r}-\tilde{\gamma}_{r}\right| & \leq\left\|\mathbf{C} \mathbf{V}_{2}-\tilde{\mathbf{C}} \tilde{\mathbf{V}}_{2} \mathbf{Q}^{H} \mathbf{R}\right\|_{2} \\
& \leq\left\|(\mathbf{C}-\tilde{\mathbf{C}}) \tilde{\mathbf{V}}_{2} \mathbf{Q}^{H} \mathbf{R}\right\|_{2}+\|\mathbf{C} \mathbf{T}\|_{2} \\
& \leq\|\mathbf{C}-\tilde{\mathbf{C}}\|_{2}+\|\mathbf{C}\|_{2}\|\mathbf{T}\|_{2} \\
& \leq \delta+\frac{3 \varepsilon}{\sigma_{r-1}-\sigma_{r}}\|\mathbf{C}\|_{2},
\end{aligned}
$$

was den Beweis abschließt.

Danksagung: Vorliegender Beitrag basiert auf Teilaspekten der Dissertationsschrift des Erstautors [19]. Besonderer Dank gilt in diesem Zusammenhang Herrn Professor Klaus Meerkötter (Universität Paderborn) für seine inhaltlichen und typographischen Verbesserungsvorschläge. Die Autoren danken ferner Herrn Dr. Daniel Muschick für seine zahlreichen Korrekturvorschläge zur vorliegenden Arbeit, sowie den anonymen Gutachtern.

\section{Literatur}

1. Björck, Å.; Golub, G. H.: Numerical Methods for Computing Angles Between Linear Subspaces. In: Mathematics of Computation 27 (1973), Nr. 123, S. 579-594.

2. Bunse-Gerstner, A.; Byers, R.; Mehrmann, V.; Nichols, N. K.: Numerical Computation of an Analytic Singular Value Decomposition of a Matrix Valued Function. In: Numerische Mathematik 60 (1991), Nr. 1, S. 1-39.

3. Dayawansa, W. P.; Ghosh, B. K.; Martin, C.; Wang, X.: A Necessary and Sufficient Condition for the Perspective Observability Problem. In: System \& Control Letters 25 (1995), S. 159-166.

4. Dirr, G.; Helmke, U.; Jordan, J.: Control and Observation of the Matrix Riccati Differential Equation. In: Ghosh, B. (Hrsg.); Martin, C. (Hrsg.); Zhou, Y. (Hrsg.): Emergent Problems in Nonlinear Systems and Control Bd. 393. Springer Berlin Heidelberg, 2009, S. 169-184.

5. Eising, R.: Between Controllable and Uncontrollable. In: Systems \& Control letters 4 (1984), Nr. 5, S. 263-264.

6 Entwickelt man die rechte Seite in eine Taylorreihe, so erhält man die asymptotische Abschätzung $\|\mathbf{T}\|_{2} \leq \frac{\sqrt{2} \varepsilon}{\sigma_{r-1}-\sigma_{r}}+\mathcal{O}\left(\varepsilon^{2}\right)$. 
6. Ghosh, B. K.; Jankovic, M.; Wu, Y. T.: Perspective Problems in System Theory and its Application to Machine Vision. In: Journal of Mathematical Systems, Estimation and Control 4 (1994), Nr. 1, S. 3-38.

7. Ghosh, B. K.; Loucks, E. P.: An Introduction to Perspective Observability and Recursive Identification Problems in Machine Vision. In: Proceedings of the 33rd IEEE Conference on Decision and Control Bd. 4, 1994, S. 3229-3234.

8. Hamdan, A. M. A.; Nayfeh, A. H.: Measures of Modal Controllability and Observability for First- and Second-Order Linear Systems. In: Journal of Guidance, Control, and Dynamics 12 (1989), Nr. 3, S. 421-428.

9. Konno, A.; Uchiyama, M.; Murakami, M.: ConfigurationDependent Vibration Controllability of Flexible-Link Manipulators. In: The International Journal of Robotics Research 16 (1997), Nr. 4, S. 567-576.

10. Lückel, J.; Müller, P. C.: Analyse von Steuerbarkeits-, Beobachtbarkeits- und Störbarkeitsstrukturen linearer zeitinvarianter Systeme. In: Regelungstechnik 23 (1975), Nr. 5, S. $163-171$.

11. Lindner, D. K.; Babenreier, J.; Hamdan, A. M. A.: Measures of Controllability and Observability and Residues. In: IEEE Transactions on Automatic Control 34 (1989), Nr. 6, S. 648-650.

12. Litz, L.: Modale Maße für Steuerbarkeit, Beobachtbarkeit, Regelbarkeit und Dominanz - Zusammenhänge, Schwachstellen, neue Wege. In: Regelungstechnik 31 (1983), Nr. 5, S. 148-158.

13. Liu, Z.-S.; Wang, D.-J.; Hu, H.-C.; Yu, M.: Measures of Modal Controllability and Observability in Vibration Control of Flexible Structures. In: Journal of Guidance, Control, and Dynamics 17 (1994), Nr. 6, S. 1377-1380.

14. Moschik, S.; Dourdoumas, N.: Steuerbarkeitsmaße für lineare zeitinvariante Systeme - Ein Überblick. In: International Journal Automation Austria 19 (2011), S. 1-14.

15. Moschik, S.; Stadler, M.; Dourdoumas, N.: Zur Überprüfung der perspektivischen Beobachtbarkeit zeitkontinuierlicher linearer zeitinvarianter Systeme. In: at - Automatisierungstechnik 60 (2012), Nr. 12, S. 735-742.

16. Paige, C.: Properties of Numerical Algorithms Related to Computing Controllability. In: IEEE Transactions on Automatic Control 26 (1981), Nr. 1, S. 130-138.

17. Sadri, A. M.; Wright, J. R.; Wynne, R. J.: Modelling and optimal placement of piezoelectric actuators in isotropic plates using genetic algorithms. In: Smart Materials and Structures 8 (1999), Nr. 4, S. 490

18. Schweitzer, G.: Ein aktives magnetisches Rotorlager - Auslegung und Anwendung. In: Regelungstechnik 26 (1978), Nr. 1 , S. $10-15$.

19. Seeber, R.: Strukturelle und quantitative Beurteilung der perspektivischen Beobachtbarkeit linearer zeitinvarianter Systeme, Fakultät für Elektrotechnik und Informationstechnik der Technischen Universität Graz, Diss., 2015.

20. Spetzler, M.; Narang-Siddarth, A.: Local Linear Controllability and Observability Analysis of Nonlinear Systems with Continuation Methods. In: AIAA Guidance, Navigation, and Control Conference, 2016, S. 1-21.

21. Stanchev, R.; Konigorski, U.; Votsmeier, M.: Modellbildung und Vergleich von linearen und nichtlinearen Regelungskonzepten für Drei-Wege-Abgaskatalysatoren. In: at - Automatisierungstechnik 64 (2016), Nr. 4, S. 297-311.
22. Stewart, G. W.; Sun, J.-G.: Matrix Perturbation Theory. Academic Press, 1990.

23. Tarokh, M.: Measures for Controllability, Observability and Fixed Modes. In: IEEE Transactions on Automatic Control 37 (1992), Nr. 8, S. 1268-1273.

24. Wedin, P.-Å.: Perturbation Bounds in Connection with Singular Value Decomposition. In: BIT Numerical Mathematics 12 (1972), Nr. 1, S. 99-111.

25. Weyl, H.: Das asymptotische Verteilungsgesetz der Eigenwerte linearer partieller Differentialgleichungen (mit einer Anwendung auf die Theorie der Hohlraumstrahlung). In: Mathematische Annalen 71 (1912), Nr. 4, S. 441-479.

\section{Autoreninformationen}

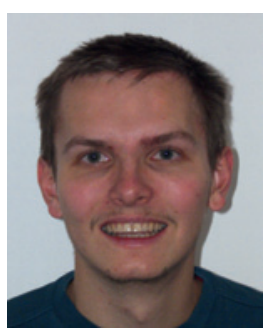

\section{Dr. techn. Richard Seeber}

Institut für Regelungs- und Automatisierungstechnik, Technische Universität Graz, Inffeldgasse 21/B/I, 8010 Graz, Österreich richard.seeber@tugraz.at

Richard Seeber ist wissenschaftlicher Mitarbeiter am Institut für Regelungs- und Automatisierungstechnik, Technische Universität Graz. Hauptarbeitsgebiete: System- und Regelungstheorie; strukturvariable Systeme.

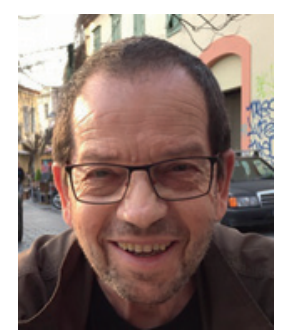

Em. O. Univ.-Prof. Dr.-Ing. Nicolaos Dourdoumas Institut für Regelungs- und Automatisierungstechnik, Technische Universität Graz, Inffeldgasse 21/B/I, 8010 Graz, Österreich nicolaos.dourdoumas@tugraz.at

Nicolaos Dourdoumas ist Emeritus am Institut für Regelungs- und Automatisierungstechnik der Technischen Universität Graz. Hauptarbeitsgebiete: System- und Regelungstheorie.

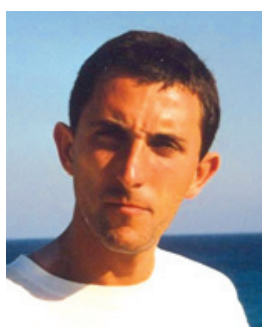

Univ.-Prof. Dr. techn. Martin Horn Institut für Regelungs- und Automatisierungstechnik, Technische Universität Graz, Inffeldgasse 21/B/I, 8010 Graz, Österreich martin.horn@tugraz.at

Martin Horn ist Vorstand des Institutes für Regelungs- und Automatisierungstechnik, Technische Universität Graz. Hauptarbeitsgebiete: Strukturvariable Systeme; Regelung vernetzter Systeme. 